\title{
Role of high-dose salvage radiotherapy for oligometastases of the localised abdominal/pelvic lymph nodes: a retrospective study
}

Makoto Ito ${ }^{1 *}$ (D), Takeshi Kodaira ${ }^{2}$, Yutaro Koide ${ }^{2}$, Takahito Okuda ${ }^{3}$, Shinichiro Mizumatsu', Yukihiko Oshima ${ }^{4}$, Arisa Takeuchi ${ }^{1}$, Toshie Mori ${ }^{1}$, Souichirou Abe ${ }^{1,2}$, Ayumi Asai ${ }^{1}$ and Kojiro Suzuki ${ }^{1}$

\begin{abstract}
Background: Abdominal/pelvic lymph node ( $L N)$ oligometastasis, a pattern of treatment failure, is observed occasionally, and radiotherapy may work as salvage therapy. The optimal prescription dose, however, is yet to be determined. This study assessed the efficacy of high-dose radiotherapy.

Methods: The medical records of 113 patients at 4 institutes were retrospectively analysed who had 1 to 5 abdominal/pelvic LN oligometastases and were treated with definitive radiotherapy between 2008 and 2018. The exclusion criteria included non-epithelial tumours, uncontrolled primary lesions, palliative intent, and re-irradiation. The prescription dose was evaluated by using the equivalent dose in 2 Gy fractions (EQD2). Patients receiving EQD2 $\geq 60$ Gy were placed into the high-dose group, and the remaining others the low-dose group. Kaplan-Meier analyses were performed to evaluate overall survival (OS), local control (LC), and progression-free survival (PFS). Univariate log-rank and multivariate Cox proportional hazards model analyses were performed to explore predictive factors. Adverse events were compared between the high-dose and low-dose groups.

Results: The primary tumour sites included the colorectum $(n=28)$, uterine cervix $(n=27)$, endometrium $(n=15)$, and ovaries $(n=10)$. The rate of 2 -year OS was $63.1 \%$, that of LC $59.7 \%$, and that of PFS $19.4 \%$. On multivariate analyses, OS were significantly associated with solitary oligometastasis (hazard ratio [HR]: 0.48, $p=0.02$ ), LC with high-dose radiotherapy (HR: 0.93, $p<0.001$ ), and PFS with long disease-free interval (HR: 0.59, $p=0.01$ ). Whereas high-dose radiotherapy did not significantly improve 2-year OS in the entire cohort (74.8\% in the high-dose vs. $52.7 \%$ in the low-dose; $p=0.08$ ), it did in the subgroup of solitary oligometastasis (88.8\% in the high-dose vs. $56.3 \%$ in the low-dose; $p=0.009$ ). As for Late grade $\geq 3$ adverse event, ileus was observed in 7 patients (6\%) and gastrointestinal bleeding in 4 (4\%). No significant association between the irradiation dose and adverse event incidence was found.
\end{abstract}

Conclusions: As salvage therapy, high-dose radiotherapy was recommendable for oligometastasis in the abdominal/pelvic LNs. For solitary oligometastasis, LC and OS were significantly better in the high-dose group.

Keywords: Oligometastasis, Oligo-recurrence, Radiotherapy, Stereotactic body radiotherapy (SBRT), Lymph node

\footnotetext{
* Correspondence: itou.makoto.292@mail.aichi-med-u.ac.jp

'Department of Radiology, Aichi Medical University Hospital, 1-1 Yazako-Karimata, Nagakute, Aichi 480-1195, Japan

Full list of author information is available at the end of the article
}

(c) The Author(s). 2020 Open Access This article is licensed under a Creative Commons Attribution 4.0 International License, which permits use, sharing, adaptation, distribution and reproduction in any medium or format, as long as you give appropriate credit to the original author(s) and the source, provide a link to the Creative Commons licence, and indicate if changes were made. The images or other third party material in this article are included in the article's Creative Commons licence, unless indicated otherwise in a credit line to the material. If material is not included in the article's Creative Commons licence and your intended use is not permitted by statutory regulation or exceeds the permitted use, you will need to obtain permission directly from the copyright holder. To view a copy of this licence, visit http://creativecommons.org/licenses/by/4.0/ The Creative Commons Public Domain Dedication waiver (http://creativecommons.org/publicdomain/zero/1.0/) applies to the data made available in this article, unless otherwise stated in a credit line to the data. 


\section{Background}

The prognosis for recurrent cancer is generally poor, and distant metastasis is especially difficult to cure. Therefore, the standard treatment of metastases is systemic chemotherapy; however, local therapy is often used to treat a small number of recurrent tumours. In 1995, oligometastases were defined as metastatic disease confined to a limited number of sites that were amenable to potentially curable local therapy such as surgery or radiotherapy [1].

Abdominal/pelvic lymph node (LN) oligometastasis is a pattern of failure that is observed occasionally, and it is usually unresectable $[2,3]$. Although radiotherapy may be the treatment choice for salvage therapy, treatment is very difficult because abdominal/pelvic LNs move randomly owing to the effect of breathing and being located close to the gastrointestinal tract. Therefore, only a few studies have evaluated the use of radiotherapy for abdominal/pelvic LN oligometastasis $[4,5]$. In particular, the optimal prescription dose for radiotherapy has not yet been clarified. The important issue is that most of them analysed cases together regardless of whether or not lesions other than LN metastasis were tightly controlled [6]. Therefore the discussions focused on local control (LC), and the survival benefits of controlling LNs were not clear. In addition, prescription doses were not uniform [6]. This makes it more difficult to determine the optimal dose for survival.

Along with the increasing use of high-precision irradiation technology, reports regarding the use of high-dose radiotherapy for oligometastasis are also increasing $[7,8]$. Recently, the SABR-COMET trial, a randomised phase 2 open-label trial, compared radiotherapy and standard of care palliative treatment for oligometastasis [9]. According to the trial, stereotactic ablative radiotherapy (SABR) was associated with improved overall survival (OS). However, treatment-related death was observed in $4.5 \%$ of patients; therefore, researchers concluded that high-dose radiotherapy should be adapted for those who would show a survival benefit. In addition, as various irradiation regions were included in the trial, further study is required to evaluate the balance between the risks and benefits of localised irradiation. Moreover, it is important to clarify the role of high-dose radiotherapy especially in locations where radiotherapy is difficult to perform, such as abdominal/pelvic LNs.

Therefore, in this study, we retrospectively reviewed the data of patients with oligometastases in the abdominal/pelvic LNs and investigated the optimal method of salvage radiotherapy. Moreover, we assessed the survival benefit of high-dose radiotherapy.

\section{Methods}

\section{Ethics approval and study design}

This retrospective observational study was approved by ethics committee, Aichi Medical University School of
Medicine in Japan (application number 2018-H211). This study was also examined and approved by Aichi Cancer Center Hospital, Toyota Memorial Hospital, and Aoyama Hospital, Japan. The study was conducted in accordance with the tenets of the Declaration of Helsinki and its subsequent amendments. Written informed consent was obtained from all patients.

\section{Patients}

Between January 2008 and April 2018, the medical records of patients treated with radiation to their abdominal/pelvic LN with a definitive intent were retrospectively reviewed from 4 institutes. In this study, patients aged 20 to 85 years who developed localised abdominal/pelvic oligometastases in 1-5 LNs after initial treatment were included. The exclusion criteria were as follows: patients with non-epithelial tumours, those with uncontrolled primary lesions, patients treated with palliative intent, those with a short follow-up ( $<3$ months), and those who received re-irradiation. Finally, a total of 113 patients were included.

The initial treatment of the primary disease was either surgery alone $(n=101)$ or definitive radiotherapy $(n=$ 12). Before undergoing salvage radiotherapy, all the patients underwent whole-body computed tomography (CT) and/or positron emission tomography-CT (PET$\mathrm{CT}$ ) to confirm that there were no oligometastases other than those in the abdominal/pelvic LNs. Owing to the presence of oligometastases after initial treatment, 91 patients underwent chemotherapy and 33 underwent concurrent chemotherapy and salvage radiotherapy.

\section{Radiotherapy}

A linear accelerator or CyberKnife (CK) was used for salvage radiotherapy. Patients were immobilised in the supine position, and a CT scan with a 1-5-mm slice thickness was obtained for treatment planning. For highprecision radiotherapy, such as intensity-modulated radiotherapy (IMRT) and stereotactic body radiotherapy (SBRT), an external vacuum-type body mould and/or a thermoplastic body mask were used for precise fixation. To avoid large displacement of the gastrointestinal tract during daily treatment, planning CT was performed with the patient in a fasting state, especially when the target was near the stomach. The clinical target volume (CTV) was defined as the gross tumour volume with a $0-0.5$ $\mathrm{cm}$ margin considering microscopic disease and the anatomical structure. The planning target volume (PTV) was defined as the CTV with a $0.3-1.0-\mathrm{cm}$ margin, considering the internal motion and the setting error in each institution. Moreover, the PTV margin was suitably adjusted to protect organs at risk (OARs). While using the linear accelerators, the abdominal/pelvic $\mathrm{LN}$ region was divided into 4 regions: para-aortic, iliac, presacral, 
and obturator. The nodal area that includes the gross tumour was defined as a prophylactic nodal area. Regarding iliac and obturator regions, the side of the tumour was delineated. In cases of 2 oligometastases or more, prophylactic nodal areas were made smaller so that the irradiation area would not be too large. Prophylactic nodal areas were expanded by $0.5 \mathrm{~cm}$ considering setup errors. The main OARs were evaluated by using the equivalent dose in 2 Gy fractions (EQD2) at $\alpha / \beta=3$, including the stomach, small bowel, large bowel, kidneys, and spinal cord. For 3-dimensional conformal radiotherapy (3DCRT), dose constraints were defined as follows: the maximum point dose was 52 Gy to the stomach and small bowel, 62 Gy to the large bowel, and 50 Gy to the spinal cord. For high-precision radiotherapy the dose constraints were defined as follows: the stomach and small bowel received a point dose of 54 Gy (V54) for $<3$ $\mathrm{cm}^{3}$, the large bowel V64 for $<3 \mathrm{~cm}^{3}$, and the kidneys a V50 of $<33 \%$. The maximum point dose to the spinal cord was $<52 \mathrm{~Gy}$.

\section{Linear accelerator}

Sixty-one patients were treated with a linear accelerator. Both the target volume and normal organ structures were contoured using treatment planning systems (either $\mathrm{XiO}$, Electa, CMS, St Louis, MO, USA or Eclipse, Varian Medical System, Palo Alto, CA, USA). All the patients were treated with $10 \mathrm{MV}$ photons, using the 3DCRT or IMRT technique. Patients treated with 3DCRT $(n=47)$ were prescribed $39.6-70 \mathrm{~Gy}$ in $15-37$ fractions to the PTV isocenter; the median dose per fraction was $2 \mathrm{~Gy}$ (range, 1.8-3 Gy) and the median EQD2 was $50 \mathrm{~Gy}$. In contrast, patients treated with IMRT $(n=14)$ received 45-70 Gy in 15-35 fractions, where the PTV was covered with a $95 \%$ dose; the median dose per fraction was 2 Gy (range, 1.8-3.5 Gy) and the median EQD2 was 61.4 Gy. More detailed data such as treatment modality and dose fractionation are shown in Supplementary Table 1. The prophylactic nodal areas received almost $70-80 \%$ of the PTV dose in both 3DCRT and IMRT. Either sequential boost or simultaneous integrated boost technics were used in patients who received prophylactic nodal irradiation with IMRT.

\section{CyberKnife}

Fifty-two patients were treated with CyberKnife. All SBRT patients were treated with CyberKnife. Ten patients were treated with less than 4Gy per fraction, and they were classified as IMRT. CK G4 and M6 (Accuray Inc., Sunnyvale $C A$, USA) and $B$ were used for SBRT. In addition to using the IRIS variable collimator, the InCise multileaf collimator was used and contributed to reduction in the treatment time for CK-M6. Generally, the tumour was followed-up by using the fiducial-less tracking capability of CK under free breathing. Using a 6-MV photon beam, all treatment plans were generated using the Multiplan treatment planning software (Accuray Inc., Sunnyvale, CA, USA). The dose was prescribed for covering the PTV, ranging from 21 to $60 \mathrm{~Gy}$, and it was fractionated 2 to 30 times with a 60 to $90 \%$ isodose line; the median dose per fraction was $6 \mathrm{~Gy}$ (range, 2-13.5 Gy) and the median EQD2 was $63.5 \mathrm{~Gy}$.

\section{Data collection and statistical analyses}

As the fractionation schedules and dose evaluation method were not standardised, the dose-volume histograms of all the cases were confirmed. Then, the prescription dose of all the cases was re-evaluated by using the EQD2 (D50\%) with $\alpha / \beta=10$. The median EQD2 for all the patients was $59.7 \mathrm{~Gy}$ (range, 40.3-101.4 Gy); using the median value as the cut-off, the high-dose group was defined as patients with EQD2 $\geq 60 \mathrm{~Gy}$ and the low-dose group was defined as those with EQD2 < $60 \mathrm{~Gy}$.

According to the Response Evaluation Criteria in Solid Tumors, the initial tumour response was evaluated based on CT scans [10]. Complete response was defined as a reduction of the lymph node to $<10 \mathrm{~mm}$ in the short axis. Partial response was defined as at least a 30\% decrease from the baseline. Progressive disease was at least a $20 \%$ increase, taking as reference the smallest state. Stable disease was defined as having neither sufficient shrinkage to qualify for partial response nor sufficient increase to qualify for progressive disease. The first follow-up visit was scheduled within 1-4.5 months (median, 2.2 months) after the end of treatment. The disease-free interval (DFI) was defined as the time from the last treatment to salvage radiotherapy. The primary endpoint was the OS. The secondary endpoints included LC, progression-free survival (PFS), and adverse events. OS was measured from the start date of salvage radiotherapy to the date of the last follow-up or death from any cause. Local control (LC) was defined as progression in the target $\mathrm{LN}$ as evaluated on CT or PET-CT images. Progression-free survival (PFS) was calculated from the start date of salvage radiotherapy until the date of disease progression or death from any cause.

The OS, LC, and PFS rates were estimated using the Kaplan-Meier method [11]. Log-rank tests were used to compare the estimates of subgroups on univariate analysis. The Cox proportional hazards model was used for multivariate analysis. $P$-values $<0.05$ were statistically significant. Factors that showed a difference with $p<0.1$ on univariate analysis were entered into the multivariate analysis. Adverse events were graded according to the National Cancer Institute Common Toxicity Criteria for Adverse Events (version 5.0), and grade $\geq 3$ events were counted. As supplementary information, events after reirradiation were collected as secondary adverse events, 
separately from the primary adverse events. Reirradiation here does not refer to the second radiotherapy in the same area as the initial treatment, which was excluded in the study, but to the one performed for oligometastasis of the abdominal/pelvic LNs. All statistical analyses were performed with EZR version 1.33 (Saitama Medical Center, Jichi Medical University, Saitama, Japan), based on the R and R commander [12].

\section{Results}

\section{Patient characteristics}

The median follow-up period for all the included 113 patients was 17.8 months (range, 3.7-109.8 months). At the time of analysis, 46 patients had died. The median follow-up time for the remaining surviving patients was 23.7 months (range, 4.0-109.8 months). The patient characteristics are listed in Table 1 and the relationship between the lymph node site and the number of lesions is shown in Supplementary Table 2. Regarding the primary site, the number of cases of colorectal and ovarian cancer was high in the high-dose group, while the number of cases of uterine cervix and endometrium cancer was high in the low-dose group. There were no patients with prostate cancer by chance. Significantly more patients were treated with SBRT and IMRT in the highdose group. Prophylactic nodal irradiation and concurrent chemotherapy were often performed with 3DCRT. The median EQD2 to the PTV in the high-dose group, the low-dose group, and all patients was 66.6 Gy (range, 60.4-101.4 Gy), 50 Gy (range, 40.3-59.8 Gy), and 59.7 Gy (range, 40.3-101.4 Gy), respectively.

\section{Treatment outcomes}

The 2-year OS, LC, and PFS rates were 63.1\% (95\% CI: 52.1-72.2\%), 59.7\% (95\% CI: 48.8-69.0\%), and $19.4 \%$ (95\% CI: $12.2-27.8 \%$ ), respectively. A total of 67 patients (59.3\%) were alive at the time of analysis: 36 were alive without disease and 31 were alive with disease. Only 3 patients died of other causes: pneumonia $(n=2)$ and owing to the adverse effects of a steroid pulse for myelitis $(n=1)$. Regarding the initial response in the entire group ( $n=113), 23$ patients showed a complete response (CR), 66 showed a partial response (PR), 19 had stable disease, and 5 had progressive disease. There was no significant difference in the CR/PR rate between the highdose and low-dose groups $(80.0 \%$ vs. $77.6 \%, p=0.82)$.

The results of univariate analysis for the OS, LC, and PFS rates are shown in Table 2. Neither the primary site nor the pathological type significantly contributed to any endpoint. A longer DFI ( $\geq 8.5$ months) was associated with significantly better 2 -year OS (72.5\% vs. $53.4 \%, p=$ $0.04)$ and PFS $(24.9 \%$ vs. $13.5 \%, p=0.01)$. The number of LNs was a prognostic factor, and solitary oligometastasis showed favourable OS $(73.3 \%$ vs. $51.3 \%, p=0.01)$ and
LC $(70.4 \%$ vs. $47.8 \%, p=0.01)$ compared with multiple metastasis. In contrast, the size of the LNs did not show apparent correlation with any parameters. Although the high-dose group had significantly better LC than the low-dose group (74.9\% vs. $45.2 \%, p<0.001)$, there was no significant difference in OS between the groups.

On multivariate analyses, solitary oligometastasis was significantly correlated with OS (HR: 0.48, 95\% CI: $0.27-$ $0.87, p=0.02$ ); high-dose radiotherapy with LC (HR: 0.93, 95\% CI: $0.90-0.96, p<0.001)$; and long DFI with PFS (HR: 0.59, 95\% CI: 0.39-0.90, $p=0.01$ ) (Table 3).

\section{Subgroup analysis for solitary oligometastasis}

High-dose radiotherapy did not significantly improve the 2-year OS on analysis of the entire cohort. However, on the subgroup analysis, solitary oligometastasis, as compared with multiple oligometastases, was a significantly favourable factor for 2-year OS $(88.8 \%$ vs. $56.3 \%, p=$ 0.009 , Fig. 1a) as well as for 2 -year LC (83.2\% vs. $54.2 \%$, $p=0.01$, Fig. 1b). The characteristics of patients with only solitary oligometastasis are shown in Supplementary Table 3 . No significant bias in the important background factors seemed to contribute to OS on univariate analysis. Furthermore, another subgroup analysis using more homogeneous patients was performed. The analysis included the patients who only had a solitary lymph node metastasis in the para-aortic site. The patients were excluded if the size of the LN was larger than $3 \mathrm{~cm}$ and/ or they received greater than $10 \mathrm{~Gy}$ per fraction. The remaining 35 patients were placed into a high-dose group of 17 patients or a low-dose group of 18 patients. Though the difference was not significant, the high-dose radiotherapy group showed a favourable outcome for 2year OS $(93.8 \%$ vs. $56.5 \%, p=0.09)$ as well as for 2 -year LC $(85.2 \%$ vs. $61.6 \%, p=0.10)$.

\section{Adverse events}

No significant difference was found in the incidence of grade $\geq 3$ adverse events between the high-dose and lowdose groups. Of acute primary adverse events, grade 3 diarrhoea, was observed in 3 patients (3\%), stomachache in 1 patient (1\%), and loss of appetite in 1 patient (1\%). No grade $\geq 4$ primary adverse events or grade $\geq 3$ secondary adverse events were observed.

As for late primary adverse events, grade 3 ileus developed in 7 patients (6\%), with $4(3 \%)$ in the highdose group and $3(3 \%)$ in the low-dose group. Of the 7 patients, $6(86 \%)$ received prophylactic nodal irradiation. Three of them (43\%) were diagnosed with ileus and underwent elective bowel resection or synectenterotomy. Although 2 of them (29\%) received reirradiation, ileus as a secondary adverse event was not observed. Grade $\geq 3$ gastrointestinal bleeding was observed in 4 patients (4\%): in two patients as a primary 
Table 1 Patient characteristics

\begin{tabular}{|c|c|c|c|c|}
\hline Characteristic & & High-dose group $(n=55)$ & Low-dose group $(n=58)$ & All cases $(n=113)$ \\
\hline \multirow[t]{2}{*}{ Age (years) } & Median & 67 & 62.5 & 65 \\
\hline & Range & $49-83$ & $36-83$ & $36-83$ \\
\hline \multirow[t]{2}{*}{ Gender } & Male & 24 & 21 & 45 \\
\hline & Female & 31 & 37 & 68 \\
\hline \multirow[t]{3}{*}{ Performance status } & 0 & 35 & 33 & 68 \\
\hline & 1 & 18 & 24 & 42 \\
\hline & 2 & 2 & 1 & 3 \\
\hline \multirow[t]{6}{*}{ Primary site } & Colorectum & 20 & 8 & 28 \\
\hline & Uterine cervix & 9 & 18 & 27 \\
\hline & Endometrium & 5 & 10 & 15 \\
\hline & Ovary & 8 & 2 & 10 \\
\hline & Urothelial & 4 & 4 & 8 \\
\hline & Others & 9 & 16 & 25 \\
\hline \multirow[t]{3}{*}{ Histopathology } & Adenocarcinoma & 41 & 31 & 72 \\
\hline & Squamous cell carcinoma & 10 & 23 & 33 \\
\hline & Others & 4 & 4 & 8 \\
\hline \multirow[t]{2}{*}{ Initial category } & T-category 1:2:3:4 & $13: 20: 18: 4$ & 18:13:19:8 & $31: 33: 37: 12$ \\
\hline & $\mathrm{N}$-category positive & 32 & 32 & 64 \\
\hline \multirow[t]{2}{*}{ Duration from initial diagnosis (months) } & Median & 25.4 & 22.4 & 24.1 \\
\hline & Range & $2.9-102.4$ & $1.9-100.4$ & $1.9-102.4$ \\
\hline \multirow[t]{2}{*}{ DFI (months) } & Median & 8.7 & 8.3 & 8.5 \\
\hline & Range & $0.6-64.4$ & $0.5-86.6$ & $0.5-86.6$ \\
\hline \multirow[t]{2}{*}{ Radiation therapy method } & 3DCRT & 12 & 35 & 47 \\
\hline & SBRT\&IMRT & 43 & 23 & 66 \\
\hline \multirow[t]{4}{*}{ LN site } & Para-aortic & 29 & 43 & 72 \\
\hline & Iliac & 8 & 10 & 18 \\
\hline & Presacral & 9 & 3 & 12 \\
\hline & Obturator & 9 & 2 & 11 \\
\hline \multirow[t]{2}{*}{ Extra-regional LN for primary site } & no & 37 & 28 & 65 \\
\hline & yes & 18 & 30 & 48 \\
\hline \multirow[t]{2}{*}{ Number of lymph node } & 1 & 33 & 28 & 61 \\
\hline & $2-5$ & 22 & 30 & 52 \\
\hline \multirow[t]{2}{*}{ LN size $(\mathrm{cm})$} & Median & 2 & 2 & 2 \\
\hline & Range & $1-5.5$ & $1-5$ & $1-5.5$ \\
\hline \multirow[t]{2}{*}{ Prophylactic nodal irradiation } & no & 40 & 29 & 69 \\
\hline & yes & 15 & 29 & 44 \\
\hline \multirow[t]{2}{*}{ Chemotherapy } & no & 15 & 7 & 22 \\
\hline & yes & 40 & 51 & 91 \\
\hline \multirow[t]{2}{*}{ Concurrent chemotherapy } & no & 43 & 37 & 80 \\
\hline & yes & 12 & 21 & 33 \\
\hline \multirow[t]{2}{*}{ EQD2 (Gy) } & Median & 66.6 & 50 & 59.7 \\
\hline & Range & $60.4-101.4$ & $40.3-59.8$ & $40.3-101.4$ \\
\hline \multirow[t]{2}{*}{ Follow-up time of entire group (months) } & Median & 20.2 & 15.9 & 17.8 \\
\hline & Range & $5.8-84.7$ & $3.7-109.8$ & $3.7-109.8$ \\
\hline
\end{tabular}


Table 1 Patient characteristics (Continued)

\begin{tabular}{lllll}
\hline Characteristic & & High-dose group $(n=55)$ & Low-dose group $(n=58)$ & All cases $(n=113)$ \\
\hline Follow-up time in surviving patients (months) & Median & 23 & 24.2 & 23.7 \\
& Range & $7.6-84.7$ & $4.0-109.8$ & $4.0-109.8$
\end{tabular}

$n$ number of patients, $L N$ lymph node, DFI disease-free interval, 3DCRT 3-dimentional conformal radiation therapy, SBRT Stereotactic Body Radiation Therapy, IMRT intensity-modulated radiation therapy, EQD2 equivalent dose in 2 Gy fraction

Table 2 Univariate analysis of prognostic factors of each endpoint

\begin{tabular}{|c|c|c|c|c|c|c|c|}
\hline \multirow[t]{2}{*}{ Factor } & & \multicolumn{2}{|c|}{$2 y-O S$} & \multicolumn{2}{|c|}{$2 y-L C$} & \multicolumn{2}{|c|}{$2 y-P F S$} \\
\hline & & $\%$ & $P$ & $\%$ & $P$ & $\%$ & $P$ \\
\hline \multirow[t]{2}{*}{ Age (years) } & $<66(n=57)$ & 56.6 & 0.21 & 50.8 & 0.04 & 16.2 & 0.150 \\
\hline & $\geq 66(n=56)$ & 71.3 & & 69.2 & & 22.5 & \\
\hline \multirow[t]{2}{*}{ Gender } & Male $(n=45)$ & 55.1 & 0.11 & 59.1 & 0.42 & 21.1 & 0.97 \\
\hline & Female $(n=68)$ & 68.2 & & 60.3 & & 17.8 & \\
\hline \multirow[t]{2}{*}{ Performance status } & $0(n=68)$ & 69.2 & 0.49 & 61.6 & 0.97 & 19.1 & 0.93 \\
\hline & $1-2(n=45)$ & 54.5 & & 56.7 & & 19.9 & \\
\hline \multirow[t]{3}{*}{ Primary site } & Colorectum & 73.5 & 0.49 & 64.1 & 0.79 & 21.9 & 0.96 \\
\hline & Uterine cervix & 88.4 & & 60.7 & & 25.8 & \\
\hline & Endometrium & 54.4 & & 59.3 & & 23.3 & \\
\hline \multirow[t]{2}{*}{ Pathological type } & Adenocarcinoma & 66.1 & 0.86 & 59.7 & 0.37 & 19.4 & 0.76 \\
\hline & Squamous cell carcinoma & 67.2 & & 60.4 & & 23.5 & \\
\hline \multirow[t]{2}{*}{ Initial T-category } & $\mathrm{T} 1-2(n=64)$ & 68.7 & 0.07 & 64.2 & 0.61 & 22.9 & 0.69 \\
\hline & T3-4 $(n=49)$ & 56.0 & & 54.7 & & 16.2 & \\
\hline \multirow[t]{2}{*}{ Initial N-category } & $N$ negative $(n=49)$ & 66.6 & 0.09 & 64.2 & 0.72 & 23.8 & 0.61 \\
\hline & $\mathrm{N}$ positive $(n=64)$ & 60.2 & & 56.1 & & 16.6 & \\
\hline \multirow[t]{2}{*}{ Duration from initial diagnosis (months) } & $<24.1(n=57)$ & 60.9 & 0.94 & 63.8 & 0.43 & 20.7 & 0.59 \\
\hline & $\geq 24.1(n=56)$ & 63.9 & & 54.9 & & 18.0 & \\
\hline \multirow[t]{2}{*}{ DFI (months) } & $<8.5(n=57)$ & 53.4 & 0.04 & 52.4 & 0.30 & 13.5 & 0.01 \\
\hline & $\geq 8.5(n=56)$ & 72.5 & & 66.0 & & 24.9 & \\
\hline \multirow[t]{2}{*}{ Radiation therapy method } & 3DCRT $(n=47)$ & 56.1 & 0.49 & 50.9 & 0.01 & 26.0 & 0.82 \\
\hline & IMRT\&SBRT $(n=66)$ & 67.9 & & 64.9 & & 14.5 & \\
\hline \multirow[t]{2}{*}{ Extra-regional LN for primary site } & no $(n=65)$ & 65.5 & 0.07 & 60.6 & 0.92 & 21.9 & 0.45 \\
\hline & yes $(n=48)$ & 59.7 & & 57.7 & & 15.3 & \\
\hline \multirow[t]{2}{*}{ Number of LN } & $1(n=61)$ & 73.3 & 0.01 & 70.4 & 0.01 & 25.1 & 0.08 \\
\hline & $2-5(n=52)$ & 51.3 & & 47.8 & & 12.9 & \\
\hline \multirow[t]{2}{*}{ LN size $(\mathrm{cm})$} & $<2(n=62)$ & 71.6 & 0.17 & 66.0 & 0.06 & 18.1 & 0.68 \\
\hline & $\geq 2(n=51)$ & 52.0 & & 52.4 & & 20.7 & \\
\hline \multirow[t]{2}{*}{ Prophylactic nodal irradiation } & no $(n=69)$ & 63.7 & 0.69 & 63.3 & 0.41 & 15.8 & 0.80 \\
\hline & yes $(n=44)$ & 61.4 & & 54.9 & & 28.2 & \\
\hline \multirow[t]{2}{*}{ Concurrent chemotherapy } & no $(n=80)$ & 63.5 & 0.92 & 58.8 & 0.69 & 11.0 & 0.20 \\
\hline & yes $(n=33)$ & 59.5 & & 60.8 & & 36.1 & \\
\hline \multirow[t]{2}{*}{ EQD2 (Gy) } & $<60(n=58)$ & 52.7 & 0.08 & 45.2 & $<0.001$ & 21.1 & 0.87 \\
\hline & $\geq 60(n=55)$ & 74.8 & & 74.9 & & 17.5 & \\
\hline
\end{tabular}


Table 3 Multivariate analysis of each endpoint

\begin{tabular}{|c|c|c|c|c|c|c|c|c|c|c|}
\hline \multirow[t]{2}{*}{ Factor } & & \multicolumn{3}{|l|}{ OS } & \multicolumn{3}{|l|}{$\underline{L C}$} & \multicolumn{3}{|l|}{ PFS } \\
\hline & & $\mathrm{HR}$ & $95 \% \mathrm{Cl}$ & $P$ & $\mathrm{HR}$ & $95 \% \mathrm{Cl}$ & $P$ & $\mathrm{HR}$ & $95 \% \mathrm{Cl}$ & $P$ \\
\hline Age (years) & $<66$ vs $\geq 66$ & & & & 0.99 & $(0.97-1.02)$ & 0.69 & & & \\
\hline Initial T-category & T1-2 vs T3-4 & 1.57 & $(0.86-2.83)$ & 0.14 & & & & & & \\
\hline Initial N-category & $\mathrm{N}$ negative vs $\mathrm{N}$ positive & 1.33 & $(0.69-2.53)$ & 0.39 & & & & & & \\
\hline DFI (months) & $<8.5$ vs $\geq 8.5$ & 0.99 & $(0.99-1.01)$ & 0.11 & & & & 0.59 & $(0.39-0.90)$ & 0.01 \\
\hline Radiation therapy method & 3DCRT vs IMRT\&SBRT & & & & 0.95 & $(0.46-1.97)$ & 0.89 & & & \\
\hline Extra-regional LN for primary site & no vs yes & 0.87 & $(0.45-1.67)$ & 0.67 & & & & & & \\
\hline Number of LN & 1 vs $2-5$ & 0.48 & $(0.27-0.87)$ & 0.02 & 0.56 & $(0.30-1.03)$ & 0.06 & 0.71 & $(0.47-1.08)$ & 0.11 \\
\hline LN size $(\mathrm{cm})$ & $<2$ vs $\geq 2$ & & & & 1.52 & $(0.83-2.80)$ & 0.18 & & & \\
\hline EQD2 (Gy) & $<60$ vs $\geq 60$ & 0.97 & $(0.95-1.01)$ & 0.09 & 0.93 & $(0.90-0.96)$ & $<0.001$ & & & \\
\hline
\end{tabular}

$H R$ hazard ratio, $C l$ confidence interval, OS overall survival rate, $L C$ local control rate, PFS progression-free survival rate, DFI disease-free interval, $3 D C R T$ 3dimentional conformal radiation therapy, IMRT intensity-modulated radiation therapy, SBRT Stereotactic Body Radiation Therapy, $L N$ lymph node, EQD2 equivalent dose in $2 \mathrm{~Gy}$ fraction

adverse event, and in the other two as secondary adverse events. The details of these 4 patients are shown in Table 4 . Of the 4 patients, 2 showed grade 3 gastrointestinal bleeding and both had received reirradiation, while the remaining 2 patients had grade 4 and grade 5 gastrointestinal bleeding, respectively, with 1 patient each in the high-dose and low-dose groups. Although both these patients were identified as having PR as the initial response, disease progression was again observed during follow-up. Chemotherapy or re-irradiation could not be administered again, and best supportive care was performed. Accordingly, the cause of serious gastrointestinal bleeding for these 2 patients was clinically interpreted to be tumour progression instead of treatmentrelated.

Re-irradiation was performed for a total of 6 patients after salvage radiotherapy: 2 patients in the high-dose group and the remaining 4 in the low-dose group. As described above, 2 of the 6 patients (33\%) presented with grade 3 late secondary adverse events after re-irradiation; both of them were in the high-dose group.

\section{Discussion}

In the current retrospective study of 113 patients with abdominal/pelvic LNs recurrent cancer and

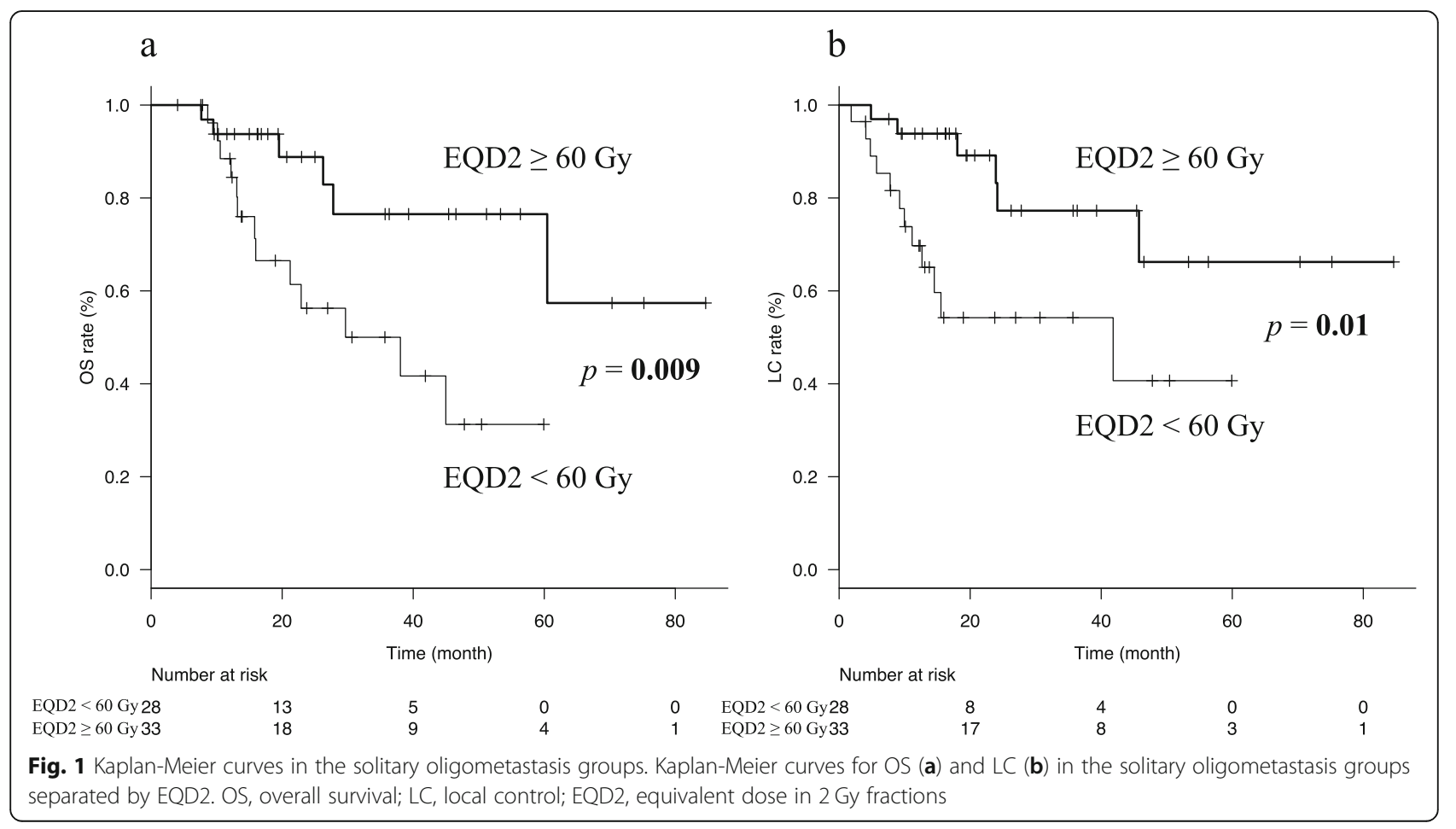


Table 4 Details of patients with Grade $\geq 3$ gastrointestinal bleeding

\begin{tabular}{|c|c|c|c|c|c|c|c|c|c|}
\hline $\mathrm{N}$ & Technique & $\begin{array}{l}\text { Prophylactic } \\
\text { nodal } \\
\text { irradiation }\end{array}$ & $\begin{array}{l}\text { Time from 1st RT to } \\
\text { re-irradiation } \\
\text { (months) }\end{array}$ & $\begin{array}{l}\text { Time from } 1 \mathrm{st} / 2 \mathrm{nd} \\
\text { RT to bleeding } \\
\text { (months) }\end{array}$ & $\begin{array}{l}{ }^{a} \text { EQD2 prescribed } \\
\text { from 1st/cumulative } \\
\text { RT (Gy) }\end{array}$ & $\begin{array}{l}\text { Location } \\
\text { of } \\
\text { bleeding }\end{array}$ & $\begin{array}{l}\text { Tumor } \\
\text { control at } \\
\text { bleeding }\end{array}$ & Grade & $\begin{array}{l}\text { Therapy for } \\
\text { bleeding }\end{array}$ \\
\hline 1 & 3DCRT & No & NA & 13.2 & 61.8 & $\begin{array}{l}\text { large } \\
\text { bowel }\end{array}$ & No & 4 & $\begin{array}{l}\text { Emergency } \\
\text { colonoscopy }\end{array}$ \\
\hline 2 & 3DCRT & Yes & 15.2 & $34.1 / 18.9$ & $44.3 / 82.3$ & $\begin{array}{l}\text { small } \\
\text { bowel }\end{array}$ & Yes & 3 & $\begin{array}{l}\text { Blood } \\
\text { transfusion }\end{array}$ \\
\hline 3 & 3DCRT & No & 9.6 & $16.8 / 7.2$ & $38.2 / 74.8$ & $\begin{array}{l}\text { large } \\
\text { bowel }\end{array}$ & Yes & 3 & $\begin{array}{l}\text { Blood } \\
\text { transfusion }\end{array}$ \\
\hline 4 & SBRT & No & NA & 9.6 & 28.2 & $\begin{array}{l}\text { small } \\
\text { bowel }\end{array}$ & No & 5 & $\begin{array}{l}\text { Best } \\
\text { supportive } \\
\text { care }\end{array}$ \\
\hline
\end{tabular}

$N$ patient number, 3DCRT 3-dimentional conformal radiation therapy, SBRT Stereotactic Body Radiotherapy, 1st First, 2nd second, EQD2 equivalent dose in 2 Gy fraction, $N A$ not applicable

${ }^{a}$ Prescribed EQD2 for location of bleeding was evaluated with $\alpha / \beta=3$.Cumulative doses were estimated by fusing the first and second dose distributions

oligometastasis from 4 institutions, the 2-year OS, LC, and PFS rates were 63.1, 59.7, and 19.4\%, respectively. Among grade $\geq 3$ late adverse events, ileus was observed in 7 patients $(6 \%)$ and gastrointestinal bleeding in 4 (4\%). The efficacy and safety were similar to those observed in previous studies [4, 5, 13-15].

In the current study, the high-dose group $(n=55)$ had significantly improved 2-year LC rates $(74.9 \%$ in the high-dose group vs. $45.2 \%$ in the low-dose group; $p<$ 0.001 ); however, improvement in 2-year OS did not reach statistical difference $(74.8 \%$ in the high-dose group vs. $52.7 \%$ in the low-dose group; $p=0.08$ ). A reason for this might be that our cohort included patients with a poor prognosis, which is a bias accompanying the retrospective nature of the study. One of the important prognostic factors is the condition of the primary lesion. Niibe et al. defined the concept of oligo-recurrence as the state in which patients with cancer have $\leq 5$ metastatic or recurrent lesions with a controlled primary lesion; they showed better prognosis than metastatic cases with active primary lesions [16]. Accordingly, in the current study, the inclusion of only patients with oligometastases was insufficient for analysis. The effect of the tumour burden was observed in $\leq 5 \mathrm{LNs}$, and the number of oligometastases seems to be a very important prognostic factor. Oehler $\mathrm{C}$ et al. defined up to 3 lymph node recurrences as oligorecurrent and retrospectively analyzed SBRT outcomes for prostate cancer after radical prostatectomy [17]. In their study, the tumor size was scrutinized and hypotheses were established as predictors of biochemical control. Nakamura et al. performed a retrospective study of 76 patients with oligo-recurrent lesions in the lungs and liver treated with SBRT. In their study, a single metastatic lesion was a significant factor of good PFS $(p=0.008)$ and a favourable OS $(p=0.053)$ [18]. Similarly, in the current study, the presence of solitary oligometastasis was associated with significantly favourable OS (HR 0.48, 95\% CI 0.27-0.87; $p=0.02$ ). Furthermore, on subgroup analysis of solitary oligometastasis, high-dose radiotherapy significantly improved LC $(p=0.01)$ and OS $(p=0.009)$. Another subgroup analysis using more homogeneous patients did not reach statistical difference, probably due to the small number of patients. The results favoured the high-dose radiotherapy group and supported the outcomes of other analyses. From these results, patients with a solitary lymph node metastasis would have a favourable prognosis, and for such patients high-dose radiotherapy might work well on OS through improving LC.

The 2-year PFS was very poor (19.4\%) regardless of the administration of prophylactic nodal irradiation. Although chemotherapy may contribute to tumour control, the precise advantage could not be determined because of the effect of bias in our study. Moreover, regarding the effectiveness of previous or adjuvant chemotherapy, there is no consensus till date $[15,18,19]$. Further individual analysis is required to clarify the efficacy of chemotherapy according to the primary disease. The DFI may be a good indicator of disease recurrence. Gandhidasan et al. reported 132 patients treated with SABR for oligometastasis [7]. In their analysis, a long DFI was significantly associated with favourable recovery from widespread disease (HR: 0.91, 95\% CI: 0.82-1.01, $p=0.041)$. Similarly, in the current study, a long DFI was associated with significantly favourable PFS (HR: 0.59, 95\% CI: 0.39-0.90, $p=0.01)$. Therefore, careful follow-up will be required especially in patients with a short DFI.

High-dose radiotherapy was as safe as low-dose radiotherapy, without any significant increase in the incidence of adverse events. However, re-irradiation especially after high-dose salvage radiotherapy might be a high-risk treatment. Of 6 patients who received re-irradiation, 2 patients $(33 \%)$ presented with grade $\geq 3$ late adverse events. Though this is a supplementary result, we propose that the execution of re-irradiation to the abdominal/pelvic LNs should be judged carefully. As the incidence of adverse events was high, we cannot 
recommend re-irradiation to the abdominal/pelvic LNs. In addition, prophylactic irradiation may be associated with the risk of ileus and its use should be considered carefully; however, as surgery is also associated with the risk of ileus, the use of prophylactic irradiation is not a major issue per se. Although a serious adverse event was observed infrequently in the current study, in the SABRCOMET trial the incidence of adverse events of grade $\geq$ 2 increased by 20 , and $4.5 \%$ of patients in the SABR group experienced a treatment-related death [9]. Accordingly, we should determine the survival benefit of using salvage radiotherapy considering the risk of adverse events.

The current study was limited by its retrospective nature and multi-institution analysis. Although our cohort did not include patients with prostate cancer, which could have led to bias, we included patients with multiple cancer types. Thus, we cannot ignore the histologyspecific differences in tumour biology. There was a bias in the patient background between the high- and lowdose groups, as well as the primary site and pathological type. In particular, the bias of the DFI and extra-regional nodes may have worked in favour of the high-dose groups. Furthermore, the treatment protocol was not constant, and the number of patients and the follow-up period were insufficient. The method of setting target volume and prescribing dose was not consistent. Especially the definition and application of prophylactic nodal irradiation were not unified. We re-evaluated with the D50 prescription, but the difference in the dose gradient from the prescription method cannot be completely eliminated. Since this study used data older than 10 years, it may be affected by treatment trends such as a hypofractionation. Accordingly, future trials should evaluate the treatment efficacy in a cohort of patients with the same tumour histology and using the same initial treatment protocol. Moreover, another limitation was the technical diversity. The margin for LNs was not standardized for all cases. In addition, the definition of the target volume varied depending on the prophylactic area. Although all the plans were reappraised by using EQD2, linear accelerators and the CK system have fundamentally different characteristics [20]. Three treatments, 3DCRT, IMRT, and SBRT, were standardized by EQD2 and evaluated together. Although a prescription dose greater than $10 \mathrm{~Gy}$ per fraction was present in only 1 case, it might not be appropriate to apply the EQD2 model to SBRT. Thus, the optimal irradiation dose should be evaluated using any one of the devices according to a single protocol.

\section{Conclusion}

Salvage radiotherapy was a feasible and safe approach for oligometastasis of the abdominal/pelvic LNs, especially in patients treated with high-dose radiotherapy (EQD2 $\geq 60$ Gy), as we observed excellent LC. For OS, the number of LNs was the most important factor, and solitary oligometastasis is an indicator of good prognosis. In patients with solitary oligometastasis, high-dose radiotherapy may play an important role in improving LC and OS. Accordingly, considering these promising results, a prospective study in a more homogeneous patient population is required to clarify the survival benefits of salvage high-dose radiotherapy.

\section{Supplementary information}

Supplementary information accompanies this paper at https://doi.org/10. 1186/s12885-020-07033-7.

Additional file 1: Supplementary Table 1. Treatment details for each patient. Supplementary Table 2. Number of lymph node metastases by site. Supplementary Table 3. Patient characteristics in the solitary oligometastasis group.

\section{Abbreviations}

3DCRT: 3-dimensional conformal radiotherapy; Cl: Confidence interval; CK: CyberKnife; CR: Complete response; CT: Computed tomography; CTV: Clinical target volume; DFI: Disease-free interval; EQD2: Equivalent dose in 2 Gy fractions; HR: Hazard ratio; IMRT: Intensity-modulated radiotherapy; LC: Local control; LN: Lymph node; OARs: Organs at risk; OS: Overall survival; PET-CT: Positron emission tomography-computed tomography; PFS: Progression-free survival; PR: Partial response; PTV: Planning target volume; SABR: Stereotactic ablative radiotherapy; SBRT: Stereotactic body radiotherapy

\section{Acknowledgements}

A part of the study results was presented at the 61st Annual Meeting of American Society of Radiation Oncology (Chicago, 2019).

We would like to thank Editage (www.editage.com) for English language editing.

\section{Authors' contributions}

$\mathrm{MI}$ conceived the study, performed all analyses, and drafted the manuscript, supported by TK, TO, and SM. YK, YO, AT, TM, SA, AA, and KS were involved in the study design and contributed significantly to the editing of the manuscript. All authors read and approved the final manuscript.

\section{Funding}

This work was partially supported by the 24th General Assembly of the Japanese Association of Medical Sciences (grant number 150-2019-3-05) and JSPS KAKENHI (grant number JP20K16741). The funding parties had no role in the design of the study, collection, analysis, interpretation of data, or in writing the manuscript.

\section{Availability of data and materials}

The datasets used and/or analysed during the current study are available from the corresponding author on reasonable request.

\section{Ethics approval and consent to participate}

All procedures involving human participants were in accordance with the ethical standards of the institutional research committee, and with the 1964 Helsinki declaration and its later amendments or comparable ethical standards. This retrospective observational study was approved by ethics committee, Aichi Medical University School of Medicine in Japan (application number 2018-H211). This study was also examined and approved by Aichi Cancer Center Hospital, Toyota Memorial Hospital, and Aoyama Hospital, Japan. The study was conducted in accordance with the tenets of the Declaration of Helsinki and its subsequent amendments. Written informed consent was obtained from all patients. 


\section{Consent for publication}

Not applicable.

\section{Competing interests}

The authors declare that they have no competing interests.

\section{Author details}

'Department of Radiology, Aichi Medical University Hospital, 1-1 Yazako-Karimata, Nagakute, Aichi 480-1195, Japan. ${ }^{2}$ Department of Radiation Oncology, Aichi Cancer Center Hospital, 1-1 Kanokoden, Chikusa-ku, Nagoya, Aichi 484-8681, Japan. ${ }^{3}$ Department of Radiation Oncology, Toyota Memorial Hospital, 1-1-1 Heiwa-cho, Toyota, Aichi 471-8513, Japan. ${ }^{4}$ Department of CyberKnife Center, Aoyama Hospital, 100-1 Kozakai-tyo-doji, Toyokawa, Aichi 441-0195, Japan.

\section{Received: 25 September 2019 Accepted: 3 June 2020}

Published online: 09 June 2020

\section{References}

1. Hellman S, Weichselbaum RR. Oligometastases. J Clin Oncol. 1995;13:8-10.

2. Lee J, Yoon HI, Rha SY, Hyung WJ, Lee YC, Lim JS, Kim HS, Koom WS. Integration of radiotherapy and chemotherapy for abdominal lymph node recurrence in gastric cancer. Clin Transl Oncol. 2017;19:1268-75.

3. Ito M, Kodaira T, Tachibana H, Tomita N, Makita C, Koide Y, Kato D, Abe T, Muro K, Tajika M, et al. Clinical results of definitive chemoradiotherapy for cervical esophageal cancer: comparison of failure pattern and toxicities between intensity-modulated radiotherapy and 3-dimensional conformal radiotherapy. Head Neck. 2017;39:2406-15.

4. Jereczek-Fossa BA, Piperno G, Ronchi S, Catalano G, Fodor C, Cambria R, Fossati Ing $\mathrm{P}$, Gherardi F, Alterio D, Zerini D, et al. Linac-based stereotactic body radiotherapy for oligometastatic patients with single abdominal lymph node recurrent cancer. Am J Clin Oncol. 2014;37:227-33.

5. Franzese C, Cozzi L, Franceschini D, D'Agostino G, Comito T, De Rose F, Navarria P, Mancosu P, Tomatis S, Fogliata A, Scorsetti M. Role of stereotactic body radiation therapy with volumetric-modulated arcs and high-intensity photon beams for the treatment of Abdomino-pelvic lymphnode metastases. Cancer Investig. 2016:34:348-54.

6. Jereczek-Fossa BA, Ronchi $\mathrm{S}$, Orecchia R. Is stereotactic body radiotherapy (SBRT) in lymph node oligometastatic patients feasible and effective? Rep Pract Oncol Radiother. 2015;20:472-83.

7. Gandhidasan S, Ball D, Kron T, Bressel M, Shaw M, Chu J, Chander S, Wheeler G, Plumridge N, Chesson B, et al. Single fraction stereotactic ablative body radiotherapy for Oligometastasis: outcomes from 132 consecutive patients. Clin Oncol (R Coll Radiol). 2018;30:178-84.

8. Fumagalli I, Bibault JE, Dewas S, Kramar A, Mirabel X, Prevost B, Lacornerie T, Jerraya $\mathrm{H}$, Lartigau $\mathrm{E}$. A single-institution study of stereotactic body radiotherapy for patients with unresectable visceral pulmonary or hepatic oligometastases. Radiat Oncol. 2012;7:164.

9. Palma DA, Olson R, Harrow S, Gaede S, Louie AV, Haasbeek C, Mulroy L, Lock M, Rodrigues GB, Yaremko BP, et al. Stereotactic ablative radiotherapy versus standard of care palliative treatment in patients with oligometastatic cancers (SABR-COMET): a randomised, phase 2, open-label trial. Lancet. 2019;393:2051-8.

10. Eisenhauer EA, Therasse P, Bogaerts J, Schwartz LH, Sargent D, Ford R, Dancey J, Arbuck S, Gwyther S, Mooney M, et al. New response evaluation criteria in solid tumours: revised RECIST guideline (version 1.1). Eur J Cancer. 2009:45:228-47.

11. Kaplan EL, Meier P. Nonparametric estimation from incomplete observations. J Am Stat Assoc. 1958;53:457-81.

12. Kanda Y. Investigation of the freely available easy-to-use software 'EZR' for medical statistics. Bone Marrow Transplant. 2013;48:452-8.

13. Bignardi M, Navarria P, Mancosu P, Cozzi L, Fogliata A, Tozzi A, Castiglioni S, Carnaghi C, Tronconi MC, Santoro A, Scorsetti M. Clinical outcome of hypofractionated stereotactic radiotherapy for abdominal lymph node metastases. Int J Radiat Oncol Biol Phys. 2011;81:831-8.

14. Corvo R, Lamanna G, Vagge S, Belgioia L, Bosetti D, Aloi D, Timon G, Bacigalupo A. Once-weekly stereotactic radiotherapy for patients with oligometastases: compliance and preliminary efficacy. Tumori. 2013;99: 159-63.

15. Alongi F, Fogliata A, Clerici E, Navarria P, Tozzi A, Comito T, Ascolese AM, Clivio A, Lobefalo F, Reggiori G, et al. Volumetric modulated arc therapy with flattening filter free beams for isolated abdominal/pelvic lymph nodes: report of dosimetric and early clinical results in oligometastatic patients. Radiat Oncol. 2012;7:204.

16. Niibe Y, Chang JY. Novel insights of oligometastases and oligo-recurrence and review of the literature. Pulm Med. 2012;2012:261096.

17. Oehler C, Zimmermann M, Adam L, Curschmann J, Sumila M, Strebel RT, Cathomas R, Li Q, Schneider U, Zwahlen DR. Predictive factors for response to salvage stereotactic body radiotherapy in oligorecurrent prostate cancer limited to lymph nodes: a single institution experience. BMC Urol. 2019;19:84.

18. Nakamura M, Hashimoto N, Mayahara H, Uezono H, Harada A, Nishikawa R, Matsuo Y, Kawaguchi H, Nishimura H. Additional chemotherapy improved local control and overall survival after stereotactic body radiation therapy for patients with oligo-recurrence. Radiat Oncol. 2018;13:75.

19. Klement RJ, Guckenberger M, Alheid H, Allgauer M, Becker G, Blanck O, Boda-Heggemann J, Brunner T, Duma M, Gerum S, et al. Stereotactic body radiotherapy for oligo-metastatic liver disease - influence of pre-treatment chemotherapy and histology on local tumor control. Radiother Oncol. 2017; 123:227-33.

20. Ito M, Kawamura T, Mori Y, Mori T, Takeuchi A, Oshima Y, Nakamura K, Aoyama T, Kaneda N, Ishiguchi T, Mizumatsu S. Dose distributions of highprecision radiotherapy treatment: a comparison between the CyberKnife and TrueBeam systems. Int J Radiat Res. 2018;16:395-402.

\section{Publisher's Note}

Springer Nature remains neutral with regard to jurisdictional claims in published maps and institutional affiliations.

\section{Ready to submit your research? Choose BMC and benefit from:}

- fast, convenient online submission

- thorough peer review by experienced researchers in your field

- rapid publication on acceptance

- support for research data, including large and complex data types

- gold Open Access which fosters wider collaboration and increased citations

- maximum visibility for your research: over $100 \mathrm{M}$ website views per year

At BMC, research is always in progress.

Learn more biomedcentral.com/submissions 